\title{
Increased efficiency of phase plate STEM using 2D detector
}

Misaki Tsubouchi ${ }^{1}$ and Hiroki Minoda ${ }^{2}$

${ }^{1}$ Tokyo University of Agriculture and Technology, United States, ${ }^{2}$ Tokyo University of Agriculture and Technology, Koganei, Tokyo, Japan

We have been developing phase plate transmission electron microscopy (PSTEM) to enhance phase contrast especially at low spatial frequency. Zernike type phase plate (ZPP), which was used to enhance the image contrast of biological materials in TEM, is used in our PSTEM. The ZPP is one of the pattered phase plate and is set in the electron illumination system of the microscope. The PP can apply a phase shift according to its pattern to the incident electron wave and can modify the phase contrast transfer function (PCTF). Since only the electrons within the small collection angle have beta to be used to realize the same optical condition as that of phase plate TEM [1,2], we can use only a small fraction of the electrons arrive onto a detector plane. Therefore, low electron efficiency was a critical problem so far.

In the present study two dimensional (2D) electron detector is used to increase electron efficiency of the PSTEM images. The 2D detector can detect the electron intensity at each pixel position and the optical condition of the electron waves depend on the pixel position. Therefore, the signal detected at each position can be made filtering depending on the pixel position to correct the optical condition. Using this procedure we can correct the PCTF of the STEM image obtained under the non-PSTEM optical condition. Figure 1 shows comparison of the image using the electron signals whose collection angle smaller than beta (a) and modified PSTEM images using the electron signals whose collection angle larger than beta (b). To construct the STEM image in (b) the PCTF was corrected by the filtering procedure. We used core-shell quantum dots dispersed on amorphous carbon film as a model sample and dark regions in STEM images correspond to the quantum dots. Intensity profiles measured along the white broken lines are below each STEM image. Figure 1(b) is very similar to Fig. 1(a). The dips in the intensity profile correspond to the quantum dots seen in (a) is larger than that in (b), but this larger dips are partly because the large noise. Considering the background noise, signal level in (b) is similar to that in (a). Therefore, this result shows that the distortion effect due to the lens aberration can be corrected by the present filtering procedure. The detected signal in Fig. 1(b) is about two orders of magnitude larger than that in Fig.1 (a) and the filtering procedure is effective. Then, we can increase the electron efficiency by this filtering procedure. 

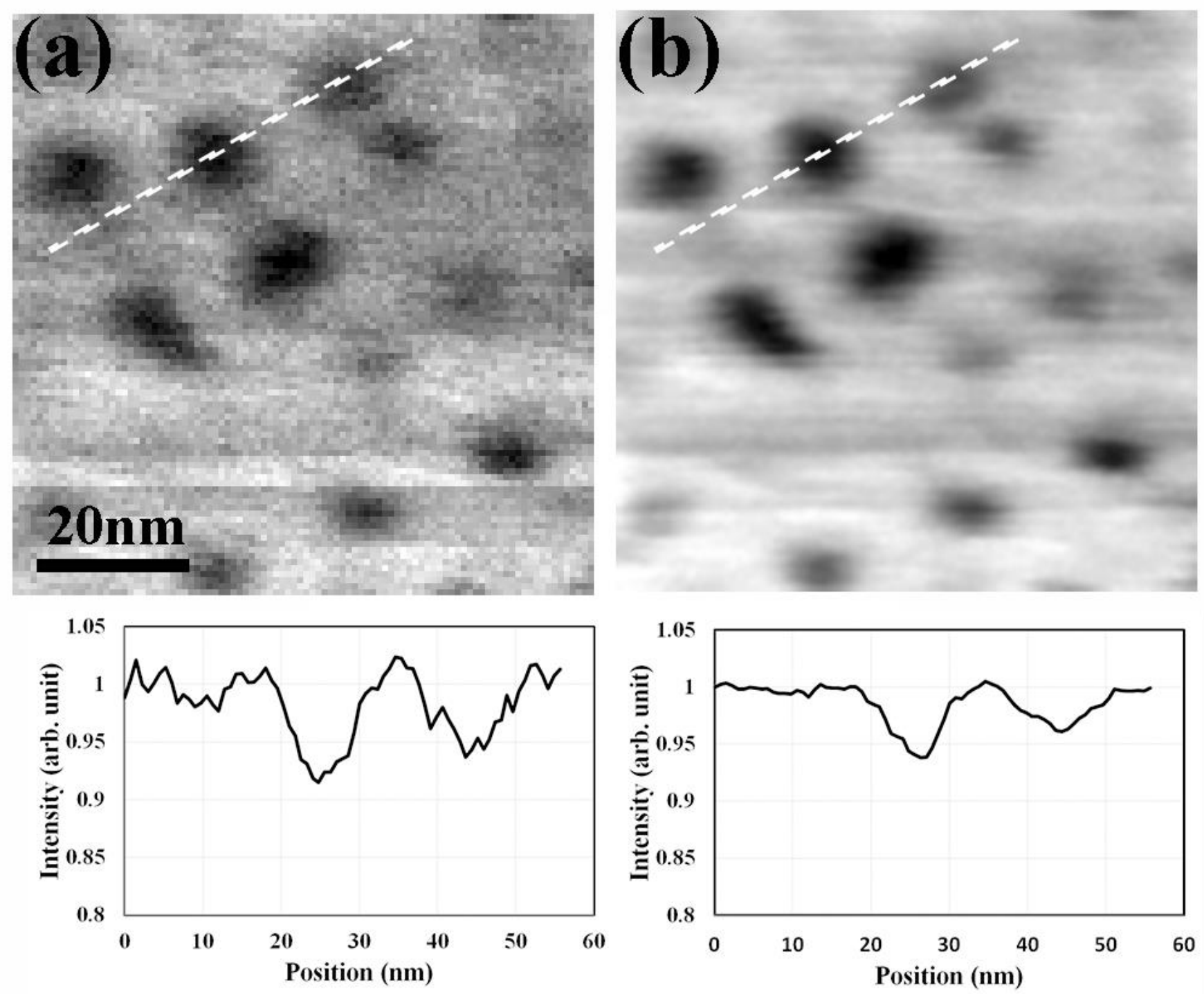

Figure 1. (a) conventional PSTEM image and (b) STEM image obtained by making the PCTF filtering procedure. Intensity profiles along the broken lines in each image were below the STEM images.

\section{References}

[1] H. Minoda, T. Tamai, H. iijima, F. Hosokawa and Y. Kondo, Microscopy 64 (2015) 181

[2] H. minoda, T. Tamai, Y. ohmori and H. Iijima, Ultramicrosocopy 182 (2017) 163. 